\title{
Compliance with Irish food-based dietary guidelines in elderly subjects recruited from rehabilitation wards and out-patient clinics in Southern region hospitals (The ELDERMET project)
}

\author{
S. E. Power ${ }^{1,2}$, P. W. O'Toole ${ }^{1,2}$, E. M. O'Connor ${ }^{1}$ and G. F. Fitzgerald ${ }^{1,2}$, \\ On Behalf Of The Em Consortium (see http://eldermet.ucc.ie For Consortium Membership) \\ ${ }^{1}$ Department of Microbiology, University College Cork, Republic of Ireland and ${ }^{2}$ Alimentary Pharmabiotic Centre, Cork, \\ Republic of Ireland
}

The elderly population are an "at-risk" group for under-nutrition which is associated with longer duration in hospitalised care as well as increased prevalence of morbidity and mortality ${ }^{(1)}$. Identifying food consumption patterns and the contribution of the main food groups to nutritional status is essential to identify those at risk of under- or over-nutrition. The aim of this study was to establish the frequency of consumption of the major food groups in two groups of elderly Irish subjects, who participated in the ELDERMET project, and to explore their compliance with the recently revised Irish food based dietary recommendations ${ }^{(2)}$.

Subjects were recruited from rehabilitation wards ( $n$ 62; aged 65-93y) and out-patient clinics ( $n$ 45; aged 67-92 y) in two Southern region hospitals. Dietary data was collected using a validated semi-quantitative, food frequency questionnaire (FFQ). Daily food group consumption was estimated and compared to Irish food-based dietary guidelines.

Over $82 \%$ of rehab and out-patient subjects complied with the recommended number of servings for breads, cereals and potatoes. In addition, both groups consumed adequate fruit and vegetables (see Table) with $51.6 \%$ of rehab and $53.3 \%$ of out-patient subjects meeting recommendations to consume $5+$ servings per day. However, poor compliance was evident for dairy-rich foods with only $14.5 \%$ of rehab and $4.4 \%$ of out-patient subjects meeting recommendations. Compliance with recommendations for low-nutrient dense foods, high in fat and/or sugar for rehab and out-patients was also particularly low in both groups at $6.5 \%$ and $13.3 \%$, respectively. In addition, $53.2 \%$ of rehab and $66.7 \%$ of out-patient subjects were classified as overweight or obese according to Body Mass Index (BMI). This high rate of overweight and obesity is in line with general healthy population trends ${ }^{(3)}$.

\begin{tabular}{llcc}
\hline \multirow{2}{*}{ Food Group } & \multicolumn{2}{c}{ Daily consumption $($ servings)* } \\
\cline { 2 - 4 } & \multicolumn{1}{c}{ Recommended } & Rehab $(\boldsymbol{n}=\mathbf{6 2})$ & Out-patients $(\boldsymbol{n}=\mathbf{4 5})$ \\
\hline Cereals, Breads, Potatoes, Rice \& Pasta & Males: 4 Females: 3 & $4.5[4.0,5.3]$ & $4.5[3.7,5.2]$ \\
Fruit \& Vegetables & $5+$ & $5.1[3.0,7.4]$ & $5.2[3.6,8.3]$ \\
Milk, Cheese \& yogurt products & 3 & $2.0[1.3,3.2]$ & $1.4[0.8,2.5]$ \\
Meat, Fish, Poultry \& Alternatives & 2 & $2.0[1.5,2.8]$ & $1.8[1.6,2.5]$ \\
Foods High in Fat and/or Sugar & Sparingly $(<3)$ & $6.2[5.0,10.3]$ & $7.5[4.3,9.7]$ \\
\hline
\end{tabular}

*median [interquartile range] number of servings per day.

In conclusion, consumption of low nutrient density foods is excessive among both rehabilitation and out-patient elderly groups. Poor compliance with the recommended intakes for dairy-rich foods may have negative implications, particularly for bone health. There is a need to explore the reasons for poor compliance with dietary recommendations among these groups and to devise strategies to address this.

1. Cuervo et al. (2008) Annals of Nutrition and Metabolism 52(4): 299.

2. Flynn MAT et al. (2012) Public Health Nutrition 15(3): 527.

3. National Adult Nutrition Survey: Summary Report (2011) available online (http://www.iuna.net) 